\title{
A HY5-COL3-COL13 regulatory chain for controlling hypocotyl elongation in Arabidopsis
}

\author{
Bin Liu ${ }^{1}$, Hong Long ${ }^{2}$, Jing Yan², Lili Ye², Qin Zhang², Hongmei Chen², Sujuan Gao², \\ Yaqin Wang ${ }^{2}$, Xiaojing Wang ${ }^{3}$, and Shulan Sun ${ }^{3}$ \\ ${ }^{1}$ Shanghai Jiao Tong University - Minhang Campus \\ ${ }^{2}$ South China Normal University \\ ${ }^{3}$ Guangdong Provincial Key Lab of Biotechnology for Plant Development
}

May 11, 2020

\begin{abstract}
CONSTANS-LIKE (COL) family members are commonly implicated in light signal transduction during early photomorphogenesis. However, some of their functions remain unclear. Here, we propose a role for COL13 in hypocotyl elongation in Arabidopsis thaliana. We found that COL13 RNA accumulates at high levels in hypocotyls and that a disruption in the COL13 function via a T-DNA insertion or RNAi led to the formation of longer hypocotyls of Arabidopsis seedlings under red light. On the contrary, overexpression of COL13 resulted in the formation of shorter hypocotyls. Using various genetic, genomic, and biochemical assays, we proved that another COL protein, COL3, directly binds to the promoter of COL13, and the promoter region of COL3 was targeted by the transcription factor LONG HYPOCOTYL 5 (HY5), to form an HY5-COL3-COL13 regulatory chain for regulating hypocotyl elongation under red light. Additionally, further study demonstrated that COL13 interacts with COL3, and COL13 promotes the interaction between COL3 and CONSTITUTIVE PHOTOMORPHOGENIC1 (COP1), suggesting a possible COP1-dependent COL3-COL13 feedback pathway. Our results provide new information regarding the gene network in mediating hypocotyl elongation.
\end{abstract}

\section{Introduction}

Light is one of the most important environmental cues influencing the early stages of post-germination plant development (Kami, Lorrain, Hornitschek, \& Fankhauser, 2010; Olle \& Viršile, 2013; Wu, Cameron, Ljung, \& Spalding, 2010). Light-grown seedlings exhibit a developmental response termed photomorphogenesis, resulting in short hypocotyls and expanded green cotyledons. In contrast, dark-grown seedlings are characterized by long hypocotyls and unexpanded etiolated cotyledons; this process is called skotomorphogenesis (Josse \& Halliday, 2008; McNellis \& Deng, 1995; Smith, 2000). As a central light signal repressor, the RING finger protein CONSTITUTIVE PHOTOMORPHOGENIC1 (COP1) is involved in many light-regulated responses and is responsible for the ubiquitination and degradation of several positive transcription factors in the dark (Dornan et al., 2004; Duek, Elmer, van Oosten, \& Fankhauser, 2004; Lau \& Deng, 2012; Osterlund, Hardtke, Wei, \& Deng, 2000; Seo, Watanabe, Tokutomi, Nagatani, \& Chua, 2004; Seo et al., 2003). For example, COP1 interacts with ELONGATED HYPOCOTYL 5 (HY5), which is a positive regulator under far-red, red, blue, and UV-B light conditions (Ang et al., 1998; Delker et al., 2014; Hardtke et al., 2000). COP1 also interacts with CONSTANS-LIKE3 (COL3), which acts as a positive regulator under red light and localizes to nuclear speckles. Additionally, the col3 mutant partially suppresses the cop1 mutation, suggesting that COL3 acts genetically downstream of COP1 (Datta, Hettiarachchi, Deng, \& Holm, 2006).

The loss-of-function col3 mutant has longer hypocotyls and flowers early and exhibits a reduced number of lateral branches (Datta et al., 2006). COL3 also directly interacts with B-BOX32 (BBX32), which is 
regulated by the circadian clock to mediate flowering (Tripathi, Carvallo, Hamilton, Preuss, \& Kay, 2017). Interestingly, both COL3 and BBX32 belong to the BBX zinc finger transcription factor (TF) family, which has 32 members (Kumagai et al., 2008). This gene family is divided into five groups based on whether their respective proteins contain one or two BBX motifs and whether or not they possess a CCT domain (Khanna et al., 2009). BBX family members, some of which have been characterized (Cheng \& Wang, 2005; Graeff et al., 2016; Li et al., 2014; Park et al., 2011; Preuss et al., 2012; Wang, Guthrie, Sarmast, \& Dehesh, 2014; Xu, Jiang, Li, Holm, \& Deng, 2018; Xu et al., 2016; Yang et al., 2014), have been implicated in light signal transduction during early photomorphogenesis. The first BBX protein identified in Arabidopsisthaliana was CONSTANS (CO) (Putterill, Robson, Lee, Simon, \& Coupland, 1995). In addition to CO, 16 other CO-Like (COL) proteins have been identified, which contain one or two B-box domains at the N-terminus and a CCT domain at the $\mathrm{C}$ terminus (Cheng \& Wang, 2005). However, most of their functions remain unclear.

A previous study showed that COL3 plays multiple roles in plant development (e.g., flowering, hypocotyl elongation, and lateral root formation) (Datta et al., 2006). Although COL3 is known to interact with B-BOX32 to regulate flowering (Tripathi et al., 2017), there has been little research on how COL3 regulates hypocotyl elongation and the respective downstream pathways are uncharacterized. In the present study, we proposed a role for COL13/B-BOX11 and an HY5-COL3-COL13 regulatory chain for controlling hypocotyl growth in A. thaliana .

\section{Materials and methods}

\section{Plant materials and growth conditions}

The A. thaliana mutant col13 (GK657F04-023194) in the Columbia background (Col-0) was purchased from Germany (GABI-Kat, Max Planck Institute for Plant Breeding Research, Carl-von-Linné-Weg 10, 50829 Köln, Germany) (Rosso et al., 2003), whereas col3 was generously provided by Professor Magnus Holm (Datta et al., 2006). Col-0, Ws, and their F1 hybrids were used as wild-type controls. Seed sterilization and growth were performed as previously described (Liu et al., 2016).

\section{Hypocotyl Experiments}

The light-response assays were performed as previously described with some changes (Datta et al., 2006). Plates were treated at $4{ }^{\circ} \mathrm{C}$ for $72 \mathrm{~h}$ and then moved to white light for $10 \mathrm{~h}$ to induce uniform germination. After that, the plates were transferred to different light conditions (dark, white, red, and blue light) and incubated at $22{ }^{\circ} \mathrm{C}$ for $3-6 \mathrm{~d}$ for hypocotyl measurement. Red and blue lights were generated by light emission diodes at 670 and $470 \mathrm{~nm}$, respectively, (model E-30LED; Percival Scientific). The light intensity was approximately $100 \mu \mathrm{mol} / \mathrm{m}^{2} \mathrm{~s}$ ppfd. The hypocotyl lengths of seedlings were measured and counted using ImageJ software.

\section{Plasmid construction}

Constructs for overexpression and RNAi assays: To construct the COL13 overexpression construct, the predicted full-length COL13 cDNA was cloned and inserted into the pCAMBIA1390 vector between the Sal I and Eco RI restriction sites. To generate the COL13 -RNAi transgenic plants, two fragments of the COL13 coding sequence were amplified by PCR using primers containing Pst I (5' end) and Mlu I (3' end) restriction sites, and Hin dIII (5' end) and Bam HI (3' end) restriction sites. The two fragments were inserted into the pRNAi- 0 vector in reverse orientation.

Constructs for GUS-staining assays: To construct the pCOL13 -GUS-2000 construct, a region comprising the 2000-bp promoter sequence of COL13 was cloned and inserted into the pBI121 vector between the Hin dIII and Bam HI sites. To construct the pCOL13 -GUS-2812 construct, a region comprising the 2812-bp promoter sequence of COL13 was cloned and inserted into the "1301 vector" between the Sac I and Sal I sites. To construct the pCOL3 -GUS construct, a region comprising the 967-bp promoter sequence of COL3 was cloned and inserted into the pBI101 vector between the Hind III and Xba I sites.

Constructs for yeast assays: To make the COL3 -pGBKT7,COP1 -pGBKT7, COL13 -pGBKT7, COL3 
-pGADT7,COP1 -pGADT7, and COL13 -pGADT7 constructs, theCOL3, COP1, and COL13 fragments were subcloned into the pGBKT7 vector (Gal4 DNA binding domain, Cat. No. 630489, Clontech) and pGADT7 (Gal4 activation domain, Cat. No. 630442, Clontech), as appropriate. To construct the COL3pBbidge and COL3-COL13-pBbidge constructs, the COL3 and COL13 fragments were subcloned into the pBbidge $^{\text {TM }}$ vector (Cat. No. 630404, Clontech), as appropriate. To construct the GAD-HY5 or GAD-COL3 fusion protein in yeast, the HY5 or COL3 coding sequence was subcloned into pJG4-5 with the EcoRI and XhoI sites. To construct the COL3::LacZ reporter gene, the 1119-bp COL3 promoter region was amplified from genomic DNA and cloned into the pLacZi2u vector with the HindIII and XhoI sites (Lin et al., 2007). Other LacZ reporter gene plasmids containing various truncated COL13 promoters were similarly constructed using the primers listed in Supplementary Table S1.

Constructs for GFP, CFP, and YFP assays: To construct the COL13 -GFP construct, the full-length COL13 coding region was cloned and inserted into the pBEGFP vector between the $X b a \mathrm{I}$ and $K p n \mathrm{I}$ restriction sites. To construct the COL3-CFP, COP1-CFP, COL13-CFP, COL3-YFP, COP1-YFP, and COL13-YFP constructs, the full-length coding regions of COL3 , COP1, and COL13 were cloned and inserted into the pBluescript II Phagemid vector (Y. Liu et al., 2016), as appropriate.

Constructs for Co-IP assays: To construct the 35S:COL3- HA construct, the full-length COL3 cDNA was cloned and inserted into the pCAMBIA1390-HA vector (Fang et al., 2019).

Constructs for dual-luciferase assays: Fragments of the COL3 or COL13 promoter were cloned into pGREEN0800-LUC to generate reporter vectors. A modified pBluescript vector (pBS) was used as an effector (Han et al., 2017).

The primers used are listed in Supplementary Table 1.

\section{Plant transformation}

Constructs in binary vectors were introduced into the Agrobacterium tumefaciens strain LBA4404 and transformed intoArabidopsis wild type (WT) or mutant plants by the floral-dip method (Clough \& Bent, 1998). Approximately $30 \mathrm{~T} 1$ transgenic plants for each transgene were screened on MS medium supplemented with the appropriate antibiotics, and phenotypic analyses were performed on T2 or later generations.

\section{Semi-quantitative PCR and qPCR}

Semi-quantitative PCR and qPCR analyses were performed as previously described (Zhang, Liu, et al., 2014). RNA was extracted from 5-d-old seedlings. Three biological and three technical repetitions were performed for each combination of cDNA samples and primer pairs. The primers used are listed in Supplementary Table 1.

\section{Dual-luciferase assay}

Protoplasts were isolated, and the dual-luciferase assay was performed as previously described (Han et al., 2017). Transformed protoplasts were incubated at room temperature $\left({ }^{\sim} 22\right)$ for $20-22 \mathrm{~h}$ and luciferase activities were measured using the dual-luciferase assay system (Dual-Luciferase ${ }^{\circledR}$ Reporter Assay, Promega, United States) according to the manufacturer's instructions. Firefly luciferase activity was normalized to Renilla luciferase activity. Three biological replicates were performed for all experiments.

\section{Electrophoretic mobility shift assay (EMSA)}

EMSA was performed as previously described with the LightShift Chemiluminescent EMSA Kit (Thermo Scientific, United States) (Han et al., 2017). The dual-luciferase assay mapped the COL3 binding site to a 1059 bp region of the COL13 promoter, located between 676 and 1675 bp upstream of the transcription start site (ATG) (Fig. 4b). This promoter region was used as a 5' end biotin-labeled probe and the same fragment, but unlabeled, was used as a competitor. To investigate the core-binding motif of the $1059 \mathrm{bp}$ region, a series of EMSAs involving deletions of this region were performed. We divided the $1059 \mathrm{bp}$ promoter sequence into five overlapping regions: -1675 to $-1391 \mathrm{bp}$ (probe 1), -1421 to $-1184 \mathrm{bp}$ (probe 2), -1201 to $-1040 \mathrm{bp}$ 
(probe 3), -1060 to -868 bp (probe 4), and -898 to -616 bp (probe 5). The sequence of probes is listed in Supplementary Table 2 .

\section{Yeast assays}

The yeast two-hybrid and three-hybrid assays were performed using a Clontech kit (PT3024-1 (PR973283)). For yeast two-hybrid assays, the bait vectors (pGBKT7 plus candidate genes) and prey vectors (pGAGT7 plus candidate genes) were transformed into the gold and Y187 yeast strains, respectively, and then each colony was picked for mating, and the mating solution was sprayed on SD/-Trp/-Leu/X- $\alpha$-Gal/AbA (DDO/X/A) agar plates, and positive results were confirmed by growing them on SD/-Ade/-His/-Trp/-Leu/X- $\alpha$-Gal/AbA $(\mathrm{QDO} / \mathrm{X} / \mathrm{A})$ agar plates. For the yeast three-hybrid assay, COP1-pGADT7 and COL3-COL13-pBbidge constructs were co-transformed into the gold yeast strain. Each colony was picked and grown in SD/-Leu/Met/-Trp and SD/-Leu/-Trp solutions, respectively. Normalized Miller units were calculated as a ratio of $\alpha$-galactosidase activity in yeast. For all yeast assays, we used empty vectors as controls.

\section{Co-immunoprecipitation (Co-IP)}

Co-IP was performed as previously described with some changes (Fiil, Qiu, Petersen, Petersen, \& Mundy, 2008). 35S:COL3-HA and 35S:COL13-GFP constructs were transformed into EHA105 Agrobacterium cells and then used to generate 35S:COL3-HA and 35S:COL3-HA::COL13-GFP transgenic plants. Proteins were extracted from 18-d-old seedlings. Anti-GFP used in this assay was bought from Abcam (ab290), Anti-HA used in this assay was bought from Sigma (H6908).

A $0.5 \mathrm{~g}$ sample of Arabidopsis seedlings were ground in liquid nitrogen, $1 \mathrm{ml}$ (2 volumes) of lysis buffer was added $(50 \mathrm{mM}$ Tris $\mathrm{pH} 8.0,150 \mathrm{mM} \mathrm{NaCl}$, and $1 \mathrm{mM}$ EDTA, containing 0.01 volume of $1 \times$ Protease Inhibitor Cocktail [Sigma]) or protease inhibitors ( $1 \mathrm{mM} \mathrm{PMSF}, 1 \mu \mathrm{g} / \mathrm{ml}$ aprotinin, $1 \mu \mathrm{g} / \mathrm{ml}$ leupeptin, 1 $\mu \mathrm{g} / \mathrm{ml}$ pepstatin). It was spun at $16,000 \mathrm{~g}$ for $20 \mathrm{~min}$ at $4{ }^{\circ} \mathrm{C}$, and the supernatant was transferred to a new microcentrifuge tube. Protein concentration was determined using the Bradford reagent (Bio-Rad) and bovine serum albumin was used as a standard. For immunoprecipitation (IP) reactions, $1 \mathrm{mg}$ of total protein was incubated with $5 \mu \mathrm{l}$ of polyantiserum (or pre-immune serum) in a total volume of $1 \mathrm{ml}$ of lysis buffer and incubated for $1 \mathrm{~h}$ to overnight at $4{ }^{\circ} \mathrm{C}$, and the sample was mixed by inversion. During incubation, a 40 $\mu \mathrm{l}$ wash per reaction of Protein-A-Agarose beads with $1 \mathrm{ml}$ of cold lysis buffer or phosphate-buffered saline (PBS) was used by gentle vortexing and spinning in a microcentrifuge at 12,000 $\mathrm{g}$ for $30 \mathrm{~s}$. The supernatant was carefully removed by aspiration. This process was repeated twice. A $50 \mu$ l sample of fresh lysis buffer or PBS was added. The beads were ready to be used. To precipitate the immune complexes, $50 \mu$ l of ProteinA-Sepharose (Amersham Pharmacia Biotech) slurry was added and incubated for $2 \mathrm{~h}$ to overnight at $4{ }^{\circ} \mathrm{C}$, mixing the sample head-over-tail. The beads were washed in the microcentrifuge tube by resuspension in 700 $\mu \mathrm{l}$ of lysis buffer. It was then centrifuged at $12,000 \mathrm{~g}$ for $15-30 \mathrm{~s}$ at $4{ }^{\circ} \mathrm{C}$. The effluent was discarded. Steps 7 and 8 were repeated three more times. Next, $40 \mu \mathrm{l}$ of $2 \times$ SDS sample buffer was added to the beads. The beads were gently mixed (no vortexing) to avoid spreading the beads on the column walls. The sample was heated at $95{ }^{\circ} \mathrm{C}$ for $5 \mathrm{~min}$ to ensure that the Protein-A-Sepharose complex was within the heating well. The sample was then centrifuged at $12,000 \mathrm{~g}$ for $30 \mathrm{~s}$. A sample of $10 \mu \mathrm{l}$ of the eluted immunoprecipitate was loaded on an SDS-PAGE gel (Bio-Rad). For standard western blotting analysis, $50 \mu \mathrm{g}$ of total protein was loaded. Proteins were electroblotted onto PVDF membranes (Amersham), blocked for at least $1 \mathrm{~h}$ at room temperature in PBS-Tween containing $5 \%$ (wt/vol) non-fat dried milk. Primary antibodies were added to PBS-Tween containing 5\% (wt/vol) non-fat dried milk and incubated for $1 \mathrm{~h}$. Blots were developed using an ECL Kit from Amersham Pharmacia Biotech.

\section{Histochemical GUS staining, GFP, and fluorescence resonance energy transfer (FRET) exper- iments}

Histochemical GUS staining, GFP microscopy, and FRET were performed as previously described with some modifications (Datta et al., 2006; Hou, Wu, \& Gan, 2013; Zhang, Zhang, et al., 2014). For the GUS-staining assay, the young seedlings (4 d after germination) were fixed and incubated in GUS-staining solution for $24 \mathrm{~h}$ at $37{ }^{\circ} \mathrm{C}$. The stained samples were then cleaned with $75 \%$ ethanol and observed under a dissecting 
microscope.

For the GFP assay, the fusion COL13 -GFP constructs were transformed into protoplasts for transient expression as previously described (Wu et al., 2009). Ten stable transgenic plants with COL13 -GFP were obtained using the floral-dip method. Photographs of GFP were taken using a confocal microscope Olympus.

For the FRET assay, images were acquired using an Olympus confocal microscope, and protoplasts were visualized $16 \mathrm{~h}$ after transformation. The CFP was excited by a laser diode 405 laser and YFP, by an argonion laser. The target regions were bleached with 100 iterations using an argon-ion laser at 100\% power.

\section{Statistical analysis}

Experimental data were analyzed using an ANOVA, and the statistical significance of any differences between treatments was tested using Duncan's test or t-tests. All analyses were conducted using SPSS for Windows.

\section{Results}

\section{COL13 RNA accumulates at high levels in hypocotyls}

By searching the gene expression information in the Arabidopsis Information Resource (TAIR) database (Klepikova, Kasianov, Gerasimov, Logacheva, \& Penin, 2016), we found that COL13(AT2G47890) is highly expressed in the hypocotyl. Quantitative PCR (qPCR) analysis confirmed that COL13 was expressed in most plant organs, with higher expression in the hypocotyl and stem (Fig. 1a). To determine the spatial patterns of COL13 expression in more detail, transgenic lines expressing GUS driven by the 2812 bp COL13promoter fragment were generated. As shown in Fig. 1b, GUS expression was predominantly in the hypocotyl.

\section{COL13 regulates hypocotyl elongation under red-light conditions}

To characterize the role of COL13 in plants, we obtained the corresponding Arabidopsis T-DNA insertion mutant (GK-657F04-023194, termed col13 in the following; Fig. S1a) from GABI-Kat, Max Planck Institute for Plant Breeding Research (Rosso et al., 2003). The mutation was verified by PCR (Fig. S1b), which amplified the sul gene by using the primers listed in Supplementary Table 1. To confirm that the phenotype of the col13 mutant was indeed caused by disruption of the COL13 gene, we generated COL13overexpression (OX) (Fig. 2a) and COL13 RNAi transgenic lines (Fig. 2b) for comparison.

To examine whether COL13 was involved in light responses, the WT, COL13 RNAi, and col13 seedlings were germinated and grown under different light wavelengths (white, red, blue), as well as under dark conditions. As shown in Fig. S1c, under white or red light, the COL13 RNAi and col13 seedlings had longer hypocotyls than that of the WT, whereas in blue light or dark conditions, the hypocotyl length of all seedlings was not significantly different. Therefore, our research focused on red light. For further study, COL13 OX, COL13RNAi, col13, and WT seedlings were germinated and grown under red light. We found that the COL13 OX seedlings had shorter hypocotyls than the WT seedlings under red light (Fig. 2c, d). In contrast, the COL13 RNAi and col13 seedlings had longer hypocotyls than the WT seedlings under the same conditions (Fig. 2c, e). These findings suggested that COL13 acts as a positive regulator of red-light-mediated inhibition of hypocotyl elongation.

\section{Genetic interaction and physiological characterization of hypocotyl elongation}

Given that the phyB, hy5, col3, and cop1 mutations can affect hypocotyl elongation under red-light conditions (Datta et al., 2006; Lee et al., 2007; Peter H Quail, 2002; von Arnim \& Deng, 1994), we investigated the expression of COL13 in the absence of PHYB ,COL3 , HY5, and COP1. Semi-quantitative PCR and quantitative PCR (qPCR) analysis revealed that the expression of COL13 in phyB, col3, or hy5 knockout plants was significantly reduced compared with that of the WT, whereas the expression of COL13 in the cop1 mutants was increased (Fig. 3a, b). As the expression of COL13 decreased the most in the col3 mutant, we generated transgenic lines expressing GUS under the control of the COL13 promoter with the col3mutant background. Interestingly, although the COL13 promoter was active in the hypocotyls and cotyledons in 
the WT seedlings, GUS expression was not detected in the hypocotyl in the col3 mutant background (Fig. $3 \mathrm{c})$.

To understand the functional relationship and genetic interaction between COL13 and COL3 and their role in the regulation of hypocotyl growth, we generated a col13 col3 double mutant and examined hypocotyl length under red-light conditions. Given thatcol13 was in Col-0 and col3 was in the WS background, crossing lines from different backgrounds would likely affect hypocotyl length. To reduce the effect of the background, we used the F1 hybrid of Col- $0 x$ WS as the WT. We found that, although hypocotyl length in the double-mutant col13 col3 was longer than in the WT seedlings, it was not significantly different from hypocotyl length in the single mutants, col13 or col3. (Fig. 3d). To confirm this result, we created the RNAi lines of COL13 in the col3 mutant background (Fig. 3e), and we obtained the same result as in Fig. $3 \mathrm{~d}$. Additionally, we also generated a COL13 -OX line in the col3 mutant background and showed that the hypocotyl length in this strain was similar to that of the WS and significantly shorter than that of the col3 mutant (Fig. 3e). In other words, COL13 overexpression rescued the phenotype exhibited by thecol3 mutant. Taken together, our results suggest thatCOL13 might be downstream of COL3 in the red-light-mediated signaling pathway.

\section{HY5-COL3-COL13 regulatory chain}

Based on the genetic data, the col3 hy5 double mutant behaved like the hy5 mutation (Datta et al., 2006), and COL13 might be downstream of COL3 in regulating hypocotyl elongation. We hypothesized that there would be an HY5-COL3-COL13 regulatory chain for controlling hypocotyl growth. To test this hypothesis, the HY5 and COL3 coding sequences, as well as a deletion series of the COL13 promoter, were cloned into the dual-luciferase system (Fig. 4a). As shown in Figure 4b, these dual-luciferase experiments and yeast-one hybrid assays confirmed the ability of HY5 to bind to the COL3 promoter and COL3 to bind to the COL13 promoter. Additionally, these experiments also mapped the COL3 target regions (1059 bp) to between $-1675 \mathrm{bp}$ and - $-616 \mathrm{bp}$ of the COL13 promoter (Fig. 4b). To investigate the core-binding motif of the 1059 bp region, a series of EMSAs involving deletions of this region were performed. We divided the $1059 \mathrm{bp}$ promoter sequence into five overlapping regions (Fig. S2a): -1675 to $-1391 \mathrm{bp}$ (probe 1), -1421 to $-1184 \mathrm{bp}$ (probe 2), -1201 to -1040 bp (probe 3), -1060 to -868 bp (probe 4), and -898 to -616 bp (probe 5), and showed that probe 2 (-1421 to $-1184 \mathrm{bp}$ ) was essential for binding of COL3 to the COL13 promoter (Fig. S2b). The in vivo interaction of COL3 with probe 2 was further confirmed by EMSA competition experiments that were conducted by adding excess amounts of the competitor (5-, 10-, and 25-fold higher amounts) (Fig. 4c).

\section{COL13 is located in the nucleus}

Transformation of Arabidopsis protoplasts with a construct expressing COL13-CFP indicated that COL13 is located in the nucleus (Fig. 5a), and a similar result was obtained when the root apical cells of stable COL13-GFP transgenic plants were examined (Fig. 5b).

\section{COL13 interacts with COL3, but not COP1}

According to previous reports, both COL3 and COL13 are CONSTANS (CO)-like proteins, which are related to CO (Robson et al., 2001), and as shown for COL13 above, COL3 also positively regulates red-lightmediated inhibition of hypocotyl elongation inArabidopsis (Datta et al., 2006). We also demonstrated that COL13 shares the same subcellular localization as COL3 (Fig. 5a, b). Given that COL3 can interact with BBX32 and that COL13 also belongs to the BBX zinc finger TF family, we hypothesized that COL3 might interact with COL13. This idea was supported by a two-hybrid assay revealing that COL3 interacts with COL13 protein in yeast (Fig. 6a). Next, we examined the interaction in transgenic plants expressing both COL3 and COL13 and showed that COL13 was co-immunoprecipitated with COL3 from seedling tissues (Fig. $6 \mathrm{~b})$. The phenotypes of 35S:COL3-HA and 35S:COL13-GFP transgenic plants were the same as 35S:COL3 and 35S:COL13 transgenic plants, respectively, which produced shorter hypocotyl than WT grown in the presence of red light (Fig. S3). The interaction between COL13 and COL3 was also demonstrated in plant cells in a FRET assay (Fig. 6c-f). As shown in Fig. 6c, both cyan fluorescent protein (CFP)-fused COL3 and yellow fluorescent protein (YFP)-fused COL13 were observed in the nucleus after excitation with a 405 
$\mathrm{nm}$ or $514 \mathrm{~nm}$ laser, respectively. After bleaching an area of interest with the $514 \mathrm{~nm}$ laser, YFP-COL13 fluorescence was reduced dramatically, whereas there was a clear increase in CFP-COL3 emission in the same area (Fig. 6d), indicating that FRET had occurred. The relative intensities of emissions from CFP-COL3 and YFP-COL13 in the area of interest, before and after bleaching, are shown in Fig. 6e, f.

\section{COL13 promotes interaction between COL3 and COP1}

Interestingly, although COL13 and COL3 have similar structures, containing two N-terminal tandemly repeated B-box domains and a CCT domain in the C-terminal, only COL3 can interact with COP1, and COL13 does not bind to COP1 (Fig. 6a). These results were also demonstrated by the FRET assay (Fig. S4a-h). To investigate whether COL13 influences the interaction between COP1 and COL3, we performed a yeast three-hybrid assay. In this yeast system, the COL3-COL13-pBridge construct allowed expression of COL3-BD / bait and COL13 in yeast, and COL13 was expressed only in the absence of methionine (Met). As shown in Fig. 7a, the growth of yeast carrying indicated constructs on selective medium (+Met or -Met) along with an $\alpha$-galactosidase assay that showed that COP1 and COL3 had a stronger binding activity with the expression of COL13. Based on a previous report, COP1 interacted with COL3 and inhibited the production of COL3 (Datta et al., 2006). By combining our results above, we propose a possible COP1-dependent COL3-COL13 feedback pathway (Fig. 7b), which is involved in the regulation of hypocotyl elongation.

\section{Discussion}

Light regulates photomorphogenesis in plants. A large number of genes that are involved in such photomorphogenesis processes have been identified as light receptors (Datta et al., 2006; Kircher et al., 2002; Peter H. Quail, 2002), signal transduction factors (Gangappa et al., 2013; Osterlund et al., 2000)or degradation proteins (Crocco, Holm, Yanovsky, \& Botto, 2010; Crocco et al., 2015; Delker et al., 2014). One of the immediate questions is how these genes act in a network to mediate various light-related phenotypes. It has been shown that multiple pathways are interlinked to form a gene network of photomorphogenesis (Lau \& Deng, 2012; Lee, Park, Ha, Baldwin, \& Park, 2017). Among these pathways, it is worth mentioning the ones formed by a subset of family genes termed the COL genes (Cheng \& Wang, 2005). These family of genes plays multiple roles in plant development (Datta et al., 2006; Graeff et al., 2016; Muntha et al., 2018; Tripathi et al., 2017; Wang et al., 2014). As an effort toward COL networking, we investigated the relationship between COL3 and COL13 and provided evidence that these two COLs and HY5 were connected together to form an HY5-COL3-COL13 regulatory chain that controls hypocotyl elongation in Arabidopsis(Fig. 7b). In addition, we also proposed a possible COP1-dependent COL3-COL13 feedback pathway to optimize this regulatory pathway (Fig. 7b).

Hypocotyl elongation is a genetically well-controlled process that responds to light. In Arabidopsis, several key genes are required for hypocotyl growth. Among these, COP1 is a negative regulator (McNellis, von Arnim, \& Deng, 1994), whereas HY5 and COL3 are considered to be positive (Datta et al., 2006; Hardtke et al., 2000). A previous study showed that COL3 plays a role in flowering and hypocotyl elongation (Datta et al., 2006), and COL3 is known to interact with B-BOX32 to regulate flowering (Tripathi et al., 2017). However, there has been no research on how COL3 regulates hypocotyl elongation. To explore how the COL family genes, COL3 in particular, function in the regulation of hypocotyl elongation, it will be facilitated by identifying the downstream genes. In this study, we demonstrated that COL13, whose RNA accumulated to a high level in the hypocotyl (Fig. 1), was one more positive regulator in the regulation of hypocotyl elongation under red-light conditions. For example, overexpression of COL13 or knockdown of its transcript resulted in a shorter or longer hypocotyl, respectively, (Fig. 2). To further define and characterize COL13, we analyzed the genetic interactions betweencol13 and col3. Seedlings of the col13 and col3 mutants showed reduced inhibition of hypocotyl elongation under red light (Fig. 3). Analysis of col3 col13 double mutants and COL13 transgenic plants revealed that COL3 is epistatic to COL13 concerning hypocotyl elongation (Fig. 3). Given that col3 hy5 double mutants behaved like the hy5 mutation (Datta et al., 2006), we hypothesized that there is an HY5-COL3-COL13 regulatory chain for controlling hypocotyl growth. As expected, our data showed that HY5 targeted the promoter of COL3 and COL3 directly bound to the promoter of COL13 (Fig. 4a-c), indicating that HY5, COL3, and COL13 constitute a hypocotyl regulatory pathway. 
CONSTANS-LIKE genes belong to the BBX family. Given that BBX family members are commonly involved in photomorphogenesis and that they can interact with other BBX proteins to regulate plant growth (Tripathi et al., 2017; Wang et al., 2014), COL3 may interact with other BBX proteins (for example. COL13/BBOX11) to regulate plant development under light conditions. Indeed, we provided evidence that COL13 can interact with COL3 (Fig. 6). Furthermore, we found that the expression of COL13 promoted the interaction between COP1 and COL3 (Fig. 7a). To our knowledge, COP1 is responsible for the degradation of several positive TFs, such as COL3, in the dark (Datta et al., 2006; Dornan et al., 2004; Duek et al., 2004; Lau \& Deng, 2012; Osterlund et al., 2000; Seo et al., 2004; Seo et al., 2003). Increasing the binding activity of COP1 and COL3 would lead to the degradation of COL3. As a result, there would be less COL3 to activate the expression of COL13 (Fig. 7b). The COP1-dependent COL3-COL13 feedback pathway could enrich the regulation network in hypocotyl elongation.

\section{Acknowledgements}

We are grateful to Prof. Magnus Holm (Gothenburg University, Sweden) for providing the col3 mutant seeds. This work was supported by the National Natural Science Foundation of China (31572161 and 31672188), the Youth Foundation of the National Natural Science Foundation of China (30900107), and China International Postdoctoral Program.

\section{References}

Ang, L.-H., Chattopadhyay, S., Wei, N., Oyama, T., Okada, K., Batschauer, A., \& Deng, X.-W. (1998). Molecular interaction between COP1 and HY5 defines a regulatory switch for light control of Arabidopsis development. Molecular Cell, 1 (2), 213-222.

Cheng, X. F., \& Wang, Z. Y. (2005). Overexpression of COL9, a CONSTANS-LIKE gene, delays flowering by reducing expression of CO and FT in Arabidopsis thaliana. The Plant Journal, 43 (5), 758-768.

Clough, S. J., \& Bent, A. F. (1998). Floral dip: a simplified method for Agrobacterium-mediated transformation of Arabidopsis thaliana. The Plant Journal, 16 (6), 735-743.

Crocco, C. D., Holm, M., Yanovsky, M. J., \& Botto, J. F. (2010). AtBBX21 and COP1 genetically interact in the regulation of shade avoidance. The Plant Journal, 64 (4), 551-562.

Crocco, C. D., Locascio, A., Escudero, C. M., Alabadí, D., Blázquez, M. A., \& Botto, J. F. (2015). The transcriptional regulator BBX24 impairs DELLA activity to promote shade avoidance in Arabidopsis thaliana . Nature Communications, 6 , 6202 .

Datta, S., Hettiarachchi, G., Deng, X.-W., \& Holm, M. (2006).Arabidopsis CONSTANS-LIKE3 is a positive regulator of red light signaling and root growth. The Plant Cell, 18 (1), 70-84.

Delker, C., Sonntag, L., James, G. V., Janitza, P., Ibañez, C., Ziermann, H., . . Ziegler, J. (2014). The DET1COP1-HY5 pathway constitutes a multipurpose signaling module regulating plant photomorphogenesis and thermomorphogenesis. Cell Reports, 9 (6), 1983-1989.

Dornan, D., Wertz, I., Shimizu, H., Arnott, D., Frantz, G. D., Dowd, P., . . Dixit, V. M. (2004). The ubiquitin ligase COP1 is a critical negative regulator of p53. Nature, 429 (6987), 86.

Duek, P. D., Elmer, M. V., van Oosten, V. R., \& Fankhauser, C. (2004). The degradation of HFR1, a putative bHLH class transcription factor involved in light signaling, is regulated by phosphorylation and requires COP1. Current Biology, 14 (24), 2296-2301.

Fang, M., Zhou, Z., Zhou, X., Yang, H., Li, M., \& Li, H. (2019). Overexpression of OsFTL10 induces early flowering and improves drought tolerance in Oryza sativa L. PeerJ, 7, e6422.

Fiil, B. K., Qiu, J.-L., Petersen, K., Petersen, M., \& Mundy, J. (2008). Coimmunoprecipitation (Co-IP) of nuclear proteins and chromatin immunoprecipitation (ChIP) from Arabidopsis . Cold Spring Harbor Protocols, 2008 (9), pdb. prot5049. 
Gangappa, S. N., Crocco, C. D., Johansson, H., Datta, S., Hettiarachchi, C., Holm, M., \& Botto, J. F. (2013). The Arabidopsis B-BOX protein BBX25 interacts with HY5, negatively regulating BBX22 expression to suppress seedling photomorphogenesis. The Plant Cell, 25 (4), 1243-1257.

Graeff, M., Straub, D., Eguen, T., Dolde, U., Rodrigues, V., Brandt, R., \& Wenkel, S. (2016). MicroProteinmediated recruitment of CONSTANS into a TOPLESS trimeric complex represses flowering in Arabidopsis .PLoS Genetics, 12 (3), e1005959.

Han, M., Jin, X., Yao, W., Kong, L., Huang, G., Tao, Y., . . W Wang, Y. (2017). A Mini Zinc-Finger protein (MIF) from Gerbera hybridaactivates the GASA protein family gene, GEG, to inhibit ray petal elongation. Frontiers in Plant Science, $8,1649$.

Hardtke, C. S., Gohda, K., Osterlund, M. T., Oyama, T., Okada, K., \& Deng, X. W. (2000). HY5 stability and activity in Arabidopsis is regulated by phosphorylation in its COP1 binding domain. The EMBO Journal, 19 (18), 4997-5006.

Hou, K., Wu, W., \& Gan, S.-S. (2013). SAUR36, a small auxin up RNA gene, is involved in the promotion of leaf senescence in Arabidopsis . Plant Physiology, 161 (2), 1002-1009.

Josse, E.-M., \& Halliday, K. J. (2008). Skotomorphogenesis: the dark side of light signalling. Current Biology, 18 (24), R1144-R1146.

Kami, C., Lorrain, S., Hornitschek, P., \& Fankhauser, C. (2010). Light-regulated plant growth and development. In Current Topics in Developmental Biology (Vol. 91, pp. 29-66): Elsevier.

Khanna, R., Kronmiller, B., Maszle, D. R., Coupland, G., Holm, M., Mizuno, T., \& Wu, S.-H. (2009). The Arabidopsis B-box zinc finger family. The Plant Cell, 21 (11), 3416-3420.

Kircher, S., Gil, P., Kozma-Bognár, L., Fejes, E., Speth, V., Husselstein-Muller, T., . . . Nagy, F. (2002). Nucleocytoplasmic partitioning of the plant photoreceptors phytochrome A, B, C, D, and E is regulated differentially by light and exhibits a diurnal rhythm. The Plant Cell, 14 (7), 1541-1555.

Klepikova, A. V., Kasianov, A. S., Gerasimov, E. S., Logacheva, M. D., \& Penin, A. A. (2016). A high resolution map of the Arabidopsis thaliana developmental transcriptome based on RNA-seq profiling. The Plant Journal, 88 (6), 1058-1070.

Kumagai, T., Ito, S., Nakamichi, N., Niwa, Y., Murakami, M., Yamashino, T., \& Mizuno, T. (2008). The common function of a novel subfamily of B-Box zinc finger proteins with reference to circadian-associated events in Arabidopsis thaliana . Bioscience, Biotechnology, and Biochemistry, 72 (6), 1539-1549.

Lau, O. S., \& Deng, X. W. (2012). The photomorphogenic repressors COP1 and DET1: 20 years later. Trends in Plant Science, 17 (10), 584-593.

Lee, H.-J., Park, Y.-J., Ha, J.-H., Baldwin, I. T., \& Park, C.-M. (2017). Multiple routes of light signaling during root photomorphogenesis. Trends in Plant Science, 22 (9), 803-812.

Lee, J., He, K., Stolc, V., Lee, H., Figueroa, P., Gao, Y., . . . Deng, X. W. (2007). Analysis of transcription factor HY5 genomic binding sites revealed its hierarchical role in light regulation of development. The Plant Cell, 19 (3), 731-749.

Li, F., Sun, J., Wang, D., Bai, S., Clarke, A. K., \& Holm, M. (2014). The B-box family gene STO (BBX24) in Arabidopsis thalianaregulates flowering time in different pathways. PloS One, 9 (2), e87544.

Lin, R., Ding, L., Casola, C., Ripoll, D. R., Feschotte, C., \& Wang, H. (2007). Transposase-derived transcription factors regulate light signaling in Arabidopsis . Science, 318 (5854), 1302-1305.

Liu, B., Liu, X., Yang, S., Chen, C., Xue, S., Cai, Y., . . . Ren, H. (2016). Silencing of the gibberellin receptor homolog, CsGID1a, affects locule formation in cucumber (Cucumis sativus) fruit. New Phytologist, $210(2), 551-563$. 
Liu, Y., Lai, J., Yu, M., Wang, F., Zhang, J., Jiang, J., . . . Xu, P. (2016). The Arabidopsis SUMO E3 ligase AtMMS21 dissociates the E2Fa/DPa complex in cell cycle regulation. The Plant Cell , 00439.02016.

McNellis, T. W., \& Deng, X.-W. (1995). Light control of seedling morphogenetic pattern. The Plant Cell, 7 (11), 1749.

McNellis, T. W., von Arnim, A. G., \& Deng, X.-W. (1994). Overexpression of Arabidopsis COP1 results in partial suppression of light-mediated development: evidence for a light-inactivable repressor of photomorphogenesis. The Plant Cell, 6 (10), 1391-1400.

Muntha, S. T., Zhang, L., Zhou, Y., Zhao, X., Hu, Z., Yang, J., \& Zhang, M. (2019). Phytochrome A signal transduction 1 and CONSTANS-LIKE 13 coordinately orchestrate shoot branching and flowering in leafy Brassica juncea . Plant Biotechnology Journal , 17(7): 1333-1343.

Olle, M., \& Viršile, A. (2013). The effects of light-emitting diode lighting on greenhouse plant growth and quality. Agricultural and Food Science, 22 (2), 223-234.

Osterlund, M. T., Hardtke, C. S., Wei, N., \& Deng, X. W. (2000). Targeted destabilization of HY5 during light-regulated development of Arabidopsis . Nature, 405 (6785), 462.

Park, H.-Y., Lee, S.-Y., Seok, H.-Y., Kim, S.-H., Sung, Z. R., \& Moon, Y.-H. (2011). EMF1 interacts with EIP1, EIP6 or EIP9 involved in the regulation of flowering time in Arabidopsis . Plant and Cell Physiology, 52 (8), 1376-1388.

Preuss, S. B., Meister, R., Xu, Q., Urwin, C. P., Tripodi, F. A., Screen, S. E., . . . Liu, G. (2012). Expression of the Arabidopsis thaliana BBX32 gene in soybean increases grain yield. PloS One, 7 (2), e30717.

Putterill, J., Robson, F., Lee, K., Simon, R., \& Coupland, G. (1995). The CONSTANS gene of Arabidopsis promotes flowering and encodes a protein showing similarities to zinc finger transcription factors. Cell, 80 (6), 847-857.

Quail, P. H. (2002). Phytochrome photosensory signalling networks.Nature Reviews Molecular Cell Biology, $3(2), 85-93$.

Robson, F., Costa, M. M. R., Hepworth, S. R., Vizir, I., Pinñeiro, M., Reeves, P. H., . . Coupland, G. (2001). Functional importance of conserved domains in the flowering-time gene CONSTANS demonstrated by analysis of mutant alleles and transgenic plants. The Plant Journal, 28 (6), 619-631.

Rosso, M. G., Li, Y., Strizhov, N., Reiss, B., Dekker, K., \& Weisshaar, B. (2003). An Arabidopsis thaliana TDNA mutagenized population (GABI-Kat) for flanking sequence tag-based reverse genetics. Plant Molecular Biology, 53 (1-2), 247-259.

Seo, H. S., Watanabe, E., Tokutomi, S., Nagatani, A., \& Chua, N.-H. (2004). Photoreceptor ubiquitination by COP1 E3 ligase desensitizes phytochrome A signaling. Genes $\mathscr{E}$ Development, 18 (6), 617-622.

Seo, H. S., Yang, J.-Y., Ishikawa, M., Bolle, C., Ballesteros, M. L., \& Chua, N.-H. (2003). LAF1 ubiquitination by COP1 controls photomorphogenesis and is stimulated by SPA1. Nature, 423 (6943), 995.

Smith, H. (2000). Phytochromes and light signal perception by plants - an emerging synthesis. Nature, 407 (6804), 585 .

Tripathi, P., Carvallo, M., Hamilton, E. E., Preuss, S., \& Kay, S. A. (2017). Arabidopsis B-BOX32 interacts with CONSTANS-LIKE3 to regulate flowering. Proceedings of the National Academy of Sciences of the United States of America, 114 (1), 172-177.

von Arnim, A. G., \& Deng, X.-W. (1994). Light inactivation of Arabidopsis photomorphogenic repressor COP1 involves a cell-specific regulation of its nucleocytoplasmic partitioning. Cell, 79 (6), 1035-1045. 
Wang, C.-Q., Guthrie, C., Sarmast, M. K., \& Dehesh, K. (2014). BBX19 interacts with CONSTANS to repress FLOWERING LOCUS T transcription, defining a flowering time checkpoint in Arabidopsis . The Plant Cell , 26(9): 3589-3602

Wu, F.-H., Shen, S.-C., Lee, L.-Y., Lee, S.-H., Chan, M.-T., \& Lin, C.-S. (2009). Tape-Arabidopsis Sandwicha simpler Arabidopsisprotoplast isolation method. Plant Methods, 5 (1), 16.

Wu, G., Cameron, J. N., Ljung, K., \& Spalding, E. P. (2010). A role for ABCB19-mediated polar auxin transport in seedling photomorphogenesis mediated by cryptochrome 1 and phytochrome B. The Plant Journal, 62 (2), 179-191.

Xu, D., Jiang, Y., Li, J., Holm, M., \& Deng, X. W. (2018). The B-box domain protein BBX21 promotes photomorphogenesis. Plant Physiology, 176 (3), 2365-2375.

Xu, D., Jiang, Y., Li, J., Lin, F., Holm, M., \& Deng, X. W. (2016). BBX21, an Arabidopsis B-box protein, directly activates HY5 and is targeted by COP1 for $26 \mathrm{~S}$ proteasome-mediated degradation.Proceedings of the National Academy of Sciences of the United States of America, 113(27): 7655-7660.

Yang, Y., Ma, C., Xu, Y., Wei, Q., Imtiaz, M., Lan, H., . . . Fei, Z. (2014). A zinc finger protein regulates flowering time and abiotic stress tolerance in chrysanthemum by modulating gibberellin biosynthesis. The Plant Cell , 26(5): 2038-2054.

Zhang, Y., Liu, B., Yang, S., An, J., Chen, C., Zhang, X., \& Ren, H. (2014). A cucumber DELLA homolog CsGAIP may inhibit staminate development through transcriptional repression of B class floral homeotic genes. PLoS One, 9 (3), e91804.

Zhang, Y., Zhang, X., Liu, B., Wang, W., Liu, X., Chen, C., . . . Ren, H. (2014). A GAMYB homologue CsGAMYB1 regulates sex expression of cucumber via an ethylene-independent pathway. Journal of Experimental Botany, 65 (12), 3201-3213.

\section{Author contributions}

S.S. and W.X. proposed the project. S.S., W.X., and L.B. designed the experiments. L.B., L.H., Y.J., Y.L., Z.Q., C.H., and G.S. performed the experiments. L.B. and S.S. analyzed the data. L.B. wrote the manuscript, with the participation of S.S. and W.Y.

\section{Figure legends}

Fig. 1 COL13 RNA accumulates at high levels in the hypocotyl.(a) Quantitative real-time PCR analysis of AtCOL13 transcript abundance in different tissues. R, Root; S, Stem; L, Leaf; SAM, Shoot apical meristem; H, Hypocotyl; F, Flower. (b) Activity of the COL13 promoter revealed by $\beta$-glucuronidase (GUS) staining in Arabidopsisseedlings. Bar $=100 \mathrm{~mm}$.

Fig. 2 COL13 regulates hypocotyl elongation under red-light conditions. (a) Relative expression of COL13 in Col-0 and overexpression (OX) lines. (b) Relative expression of COL13 in Col-0, T-DNA mutant (col13), and RNAi lines (R1-1 and others). (c)-(e) Phenotypic analysis of seedlings of the indicated genotypes were grown in the presence of red light. Images of representative seedlings are shown in (c). The hypocotyl lengths of the indicated genotypes were measured and are shown in (d) and (e). Error bars indicate the standard deviations $(\mathrm{n}>15)$. Asterisks indicate that hypocotyl lengths in OX9 and col13, COL13 RNAi are significantly different than that of the WT under red light $(\mathrm{P}<0.05)$.

Fig. 3 Genetic interaction and physiological characterization of hypocotyl elongation (a) Semiquantitative RT-PCR analyses of COL13 expression in phyB , col3, hy5, and cop1 mutants. (b) qRT-PCR analyses of COL13 expression in phyB, col3 , hy5, and cop1 mutants. (c) Activity of the COL13 promoter revealed by $\beta$-glucuronidase (GUS) staining in WT and col3 mutant backgrounds. (d) Hypocotyl length in WT and single- and double-mutant plants. (e) Hypocotyl length in WT and col3 plants compared to transgenic plants with COL13 RNAi or COL13 overexpression (OX) in the col3 background. Error bars 
indicate the standard deviations $(\mathrm{n}>15)$. Lower-case letters indicate significantly different data groups (hypocotyl length) for the indicated seedlings grown under red light.

Fig. 4 Analysis of the binding of HY5 to the COL3 promoter and COL3 to COL13 promoter truncations. (a) Diagram of the constructs used. The AD-HY5 or AD-COL3 fusion gene driven by the 35S promoter produces a potential effector protein, whereas the AD protein alone represents a negative control for the basal activity of the COL3 promoter or each COL13 promoter truncation. The LUC gene driven by the series of COL3 promoter or COL13 promoter truncations tests the ability of the AD-HY5 or AD-COL3 fusion protein to bind to each promoter truncation. (b) The fusion protein AD-HY5, but not AD alone, can affect LUC expression from the COL3 promoter truncations, and the fusion protein AD-COL3, but not $\mathrm{AD}$ alone, can affect LUC expression from some of the COL13 promoter truncations. (c) Electrophoretic mobility shift assay (EMSA) analysis showing the binding of COL3 to COL13 at -1421 to -1184 bp promoter (probe 2) in vitro. The black arrow indicates the binding of COL3 to the biotin-labeled COL13 promoter. The + and - represent the presence and absence of the corresponding components, respectively.

Fig. 5 Subcellular localization of COL13 (a) COL13-CFP localizes to the nucleus in protoplasts. (d) COL13-GFP localizes to the nucleus in root tip cells.

Fig. 6 COL13 interacts with COL3. (a) Yeast two-hybrid assay between COL13 and COL3. DDO, double dropout; QDO, quadruple dropout; pGADT7, prey plasmid; pGBKT7, bait plasmid. (b) Coimmunoprecipitation (Co-IP) in Arabidopsis Immunoprecipitations (IPs) were performed on proteins extracted from 10 d-old Arabidopsis seedlings grown under long-day illumination (16L: 8D) at $22{ }^{\circ} \mathrm{C}$. Leaf tissues were harvested $1 \mathrm{~h}$ after the light cycle commenced. IP was performed using an anti-HA antibody and COL13 was co-immunoprecipitated with an anti-GFP antibody. A 5\% input was used. Western blots were performed on $10 \%$ (wt/vol) precast gels (Bio-Rad). (c) COL3-CFP and COL13-YFP colocalize to the nucleus in protoplasts in the light and dark. (d-f) FRET between CFP-COL3 and YFP-COL13 analyzed by acceptor bleaching in the nucleus. The top panels in (d) show a representative pre-bleach nucleus coexpressing YFP-COL13 and CFP-COL3 excited with either a 514 or a $405 \mathrm{~nm}$ laser in light and dark, resulting in emission from YFP (yellow) or CFP (blue), respectively. The bottom panels in (d) show the same nucleus post-bleaching after excitation with a 514 or a $405 \mathrm{~nm}$ laser. The relative intensities of both YFP and CFP were measured before and after bleaching, as indicated in (e) and (f), respectively.

Fig. 7 COL13 promotes the interaction between COL3 and COP1.(a) Yeast three-hybrid analysis of COP1-COL3 interaction in the presence of COL13. Normalized Miller units were calculated as a ratio of $\alpha$ galactosidase activity in yeast. Additionally, normalized Miller units are reported separately for yeast grown on media with or without $1 \mathrm{mM}$ methionine (Met), corresponding to induction (-Met) or repression (+Met) of Met25 promoter-driven COL13 expression, respectively. Means and standard errors of the means for three biological repetitions are shown. Lower-case letters indicate significant differences in $\alpha$-galactosidase. (b) A model representing the HY5-COL3-COL13 regulatory chain and COP1-dependent COL3-COL13 feedback pathway in the regulation of hypocotyl elongation.

\section{Supporting Information}

Table S1 List of primers and their uses.

Table S2 Probes used in EMSA assay.

Fig. S1 COL13 regulates hypocotyl elongation under red-light conditions. (a) Scheme of the Arabidopsis COL13 gene (AT2G47890) showing the T-DNA insertion position. (b) Identification of the sulfonamide resistance gene (SUL) in the col13 mutant by PCR. (c) The hypocotyl lengths of the wild type (WT), COL13 RNAi, and col13 seedlings under different light conditions. Error bars indicate the standard deviations $(\mathrm{n}>15)$. Lower-case letters indicate significant differences $(\mathrm{P}<0.05)$.

Fig. S2 Electrophoretic mobility shift assay (EMSA) showing binding of COL3 to the COL13 promoter in vitro. 
Fig.S3 Hypocotyl lengths of the indicated genotypes were measured at the $5^{\text {th }}$ day. Error bars indicate SD $(\mathrm{n}>15)$. Asterisks indicate that hypocotyl lengths in 35S:COL-HA and 35S:COL13-GFP are significantly different with Col-0 under red light $(\mathrm{P}<0.05)$.

Fig. S4 COP1 can interact with COL3, but not COL13. a COL3-CFP and COP1-YFP co-localized to the nucleus in protoplasts under both light and dark conditions. b FRET between CFP-COL3 and YFPCOP1 analyzed by acceptor bleaching in the nucleus. The top panels in b show a representative pre-bleach nucleus co-expressing YFP-COP1 and CFP-COL3 excited with either a 514 or a $405 \mathrm{~nm}$ laser in light and dark, resulting in emission from YFP (yellow) or CFP (blue), respectively. The bottom panels in b show the same nucleus after bleaching following excitation with a 514 or a $405 \mathrm{~nm}$ laser. The relative intensities of both YFP and CFP were measured once before and twice after bleaching, as indicated in $\mathrm{c}$ and d. e COL13-CFP and COP1-YFP co-localized to the nucleus in protoplasts in light and dark. f FRET between CFP-COL13 and YFP-COP1 analyzed by acceptor bleaching in the nucleus. The top panels in $\mathrm{f}$ show a representative pre-bleach nucleus co-expressing YFP-COP1 and CFP-COL13 excited with either a 514 or a $405 \mathrm{~nm}$ laser in light and dark, resulting in emission from YFP (yellow) or CFP (blue), respectively. The bottom panels in $\mathrm{f}$ show the same nucleus after bleaching following excitation with a 514- or a 405-nm laser. The relative intensities of both YFP and CFP were measured once before and twice after bleaching, as indicated in $g$ and $h$. 

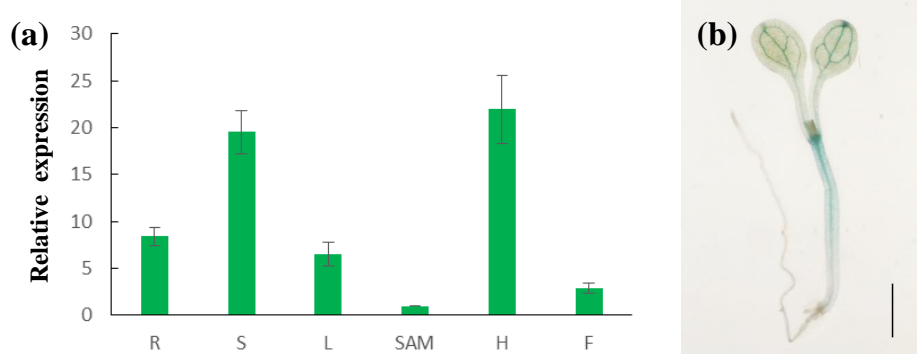

Fig.1 COL13 RNA accumulates to high levels in hypocotyl. (a) Quantitative real time-PCR analysis of $A t C O L 13$ transcript abundance in different tissues. $\mathrm{R}=$ Root, $\mathrm{S}=$ Stem, L=Leaf, SAM=Shoot apical meristem, H=Hypocotyl, F=Flower. (b) Activity of COL13 promoter revealed by $\beta$-glucuronidase (GUS) staining in Arabidopsis seedlings. Bar $=100 \mathrm{~mm}$.

\section{Hosted file}

Figures-submit.pdf available at https://authorea.com/users/297924/articles/449674-a-hy5col3-col13-regulatory-chain-for-controlling-hypocotyl-elongation-in-arabidopsis 